\title{
Digital Camera as Gloss Measurement Device
}

\author{
A. MIHÁLIK AND R. ĎURIKOVIČ
}

\begin{abstract}
Nowadays digital cameras with both high resolution and the high dynamic range (HDR) can be considered as parallel multiple sensors producing multiple measurements at once. In this paper we describe a technique for processing the captured HDR data and than fit them to theoretical surface reflection models in the form of bidirectional reflectance distribution function (BRDF). Finally, the tabular BRDF can be used to calculate the gloss reflection of the surface. We compare the captured glossiness by digital camera with gloss measured with the industry device and conclude that the results fit well in our experiments.
\end{abstract}

Mathematics Subject Classification 2000: 78A05, 68P01, 68U05

Additional Key Words and Phrases: BRDF measurements, gloss, paint reflectance.

\section{INTRODUCTION}

Manufacturers in industry often need to characterize the appearance of their products (eg. paint finish). For the characterisation of appearance used in paint, paper, plastic and textile industry, the industrial spot measurement devices, such as colorimeters or glossmeters has been developed [Dorsey et al. 2008]. Glossmeters are build according to industrial standards such as ISO 2813 [ISO2813 1994] or ASTM D523 [523-89 1999], that define the geometry of specular gloss measurement including the incidence angles of light, and optics [Hunter and Harold 1987]. Gloss is the appearance attribute of surfaces that causes them to have appearance from shiny to mat. We can measure it by focusing on the light reflected off the surface and neglecting the material structure. Industrial standards prescribe black glass as a standard for the calibration of the gloss measuring devices. Perception of the gloss is influenced by physiological and psychological aspects of the human vision. Some psychophysically-based models of surface gloss have been found by multi-dimensional scaling algorithms based on the subjective evaluation of the images [Wills et al. 2009] [James A. Ferwerda and Greenberg 2001].

Our work is devoted to the development of methods obtaining gloss values from BRDF measurements. BRDF measurements are performed using gonio-spectrophotometers. A gonio-spectrophotometer is an instrument measuring the spectral distribution of reflected radiant power as a function of angles of 


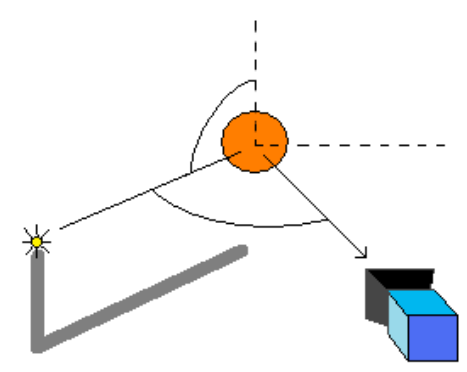

Fig. 1. A scheme of the BRDF measurement gantry

illumination and observation. The device consists of a light source aperture and a receptor aperture enabling to orient them in variety of directions to perform comprehensive measurements. Typical gonio-spectrophotometer consists of the gantry equipped with digital camera.

A gonio-spectrophotometer is defined as an instrument that measures spectral power as a function of illumination and observation directions. The light flux that is incident on the material sample comes from an emitter. After being reflected by the surface, it is captured by a detector such as a digital camera (see Figure 1). For BRDF measurements the detector is placed on hemisphere above the sample. The device requires to move the receptor aperture and the light source. Both, the direction of illumination $\Theta_{i}$ and viewing direction $\Theta_{r}$ (see Figure 2) can be varied independently within the hemisphere above the material sample. The reflected flux is recorded for each position of the source and detector aperture. Number of positions depend on the angular accuracy of recorded BRDF. Since BRDF is reciprocal, that means we get the same results when we exchange the source and receptor aperture, the number of positions could be reduced to the half.

The proposed device will take as an input the set of images with captured reflectance and estimates the BRDF as in Section 3, then the total flux reflected from both a sample and a glass standard are calculated in Section 4, finally the gloss is calculated as a ratio of total flux reflected from sample to flux reflected from the standard as in Section 4. Sometimes, the acquisition of reflectance is easier to obtain, therefore we propose the method for gloss calculation as a ratio of reflectences in Eq. 5 . 


\section{DATA ACQUISITION WITH DIGITAL CAMERA}

In our experiment we used tabular BRDF acquired by the acquisition system which requires a spherically homogenous sample of the material [Matusik et al. 2003]. The acquisition system consisted of the $1300 \times 1030$ resolution digital camera, a lamp with stable light emission output and a continuous and relatively constant radiation spectrum over the visible light range. The lamp orbited the measurement sample placed at the center of rotation; the camera and the sample was stationary. The light source moved in increments of approximately $0.5^{\circ}$ from the point opposite the camera (the sample was placed in between the camera and the light source) to the point in front of the camera. It took a total of 330 high dynamic range pictures to cover the required half circle. The CCD camera had a very linear response curve in order to derive the high dynamic measurement. Each acquired image of the sample sphere represents many BRDF samples. Essentially, each pixel of the sphere is treated as a separate BRDF measurement resulting in a huge table of BRDF measurements for given incident and reflected ray direction and for three spectral measurements: red, green and blue.

\section{GLOSS MEASUREMENTS}

Because of the complexity of full BRDF acquisition, it is more reasonable to relay on simple measurements often used in industry; those are for example, industrial spot measurement devices, such as colorimeters or glossmeters. Glossmeters are build according to industrial standards such as ISO 2813 or ASTM D523, that define the geometry of specular gloss measurement including the incidence angles and optics [Mihálik and Ďurikovič 2011]. Gloss is the appearance attribute of surfaces that causes them to have appearance from shiny to mat.

Simulation of glossmeter is used to find correspondence between the acquired BRDF model and industrial measurement scale. This correspondence can be found by variety of standardized measurements using HDR camera on multiple samples.

Measurements are measured in gloss units which describes amount of reflected light related to the amount of reflected light from a black glass standard. Those standards prescribe the measurement to be taken at angles $20^{\circ}, 60^{\circ}$ and $85^{\circ}$ to the surface normal (angles in the Figure 2 are set to $\theta_{i}=\theta_{r} \in\left\{20^{\circ}, 60^{\circ}, 85^{\circ}\right\}$ ), because these degrees of specular gloss measurements offer numerical values which are roughly linearly correlated over a range of values to perceived gloss of high-gloss, medium-gloss and low-gloss surfaces, respectively. The standards also prescribe 

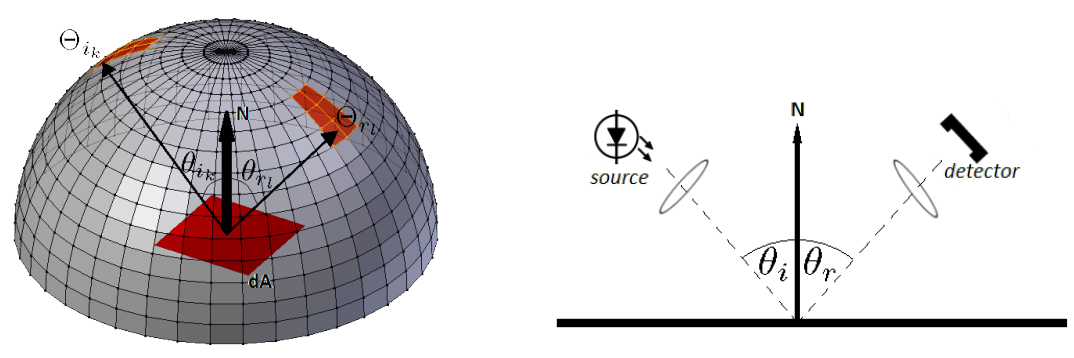

Fig. 2. Subdivided hemisphere and the geometry of the measurement. Angles $\varepsilon_{s}$ and $\varepsilon_{d}$ are angles between the surface normal and the direction to the center of source aperture and detector aperture, respectively.

sizes of source and detector apertures, see Table I.

\begin{tabular}{|l|c|c|}
\hline \multirow{2}{*}{$\begin{array}{c}\text { Angle of gloss } \\
\text { measurement }\end{array}$} & \multicolumn{2}{|c|}{ Aperture size (in degrees) } \\
\cline { 2 - 3 } & Source & Detector \\
\hline $20^{\circ}$ & $0.75 \times 2.5$ & $1.8 \times 3.6$ \\
\hline $60^{\circ}$ & $0.75 \times 2.5$ & $4.4 \times 11.7$ \\
\hline $85^{\circ}$ & $0.75 \times 2.5$ & $4.0 \times 6.0$ \\
\hline
\end{tabular}

Table I. Angular aperture sizes defined from the measured spot and the angles of the gloss measurement.

\section{GLOSS COMPUTATION}

In this section we will describe the use of measured BRDF data from Section 3 in the gloss calculation. In the simulation of gonio-spectrophotometer, the hemisphere over the sample is subdivided into small patches. Proposed virtual-goniospectrophotometer consists of light source and detector aperture. A patch belongs to the source or detector aperture (see Figure 2) iff its position is close (within adjustable tolerance which represents dimensions of apertures) to direction of the illumination $\Theta_{i}$ or observation $\Theta_{r}$, respectively.

Total flux reflected from the sample surface is computed by the sum of incident flux from the source aperture patches (from each source aperture patch in direction $\Theta_{i_{k}}$ ) reflected toward the detector aperture patches (to each detector aperture patch in direction $\Theta_{r_{l}}$ ). Thus total flux captured by the receptor aperture is computed by the numerical integration over the source and detector patches (adding the energy reflected from each patch $i_{k}$ toward each patch $r_{l}$ ).

Each patch area of the hemisphere serves us as the solid angle. Particular radiant flux is coming through all source patches, that are incident with the surface. For each 
receptor patch, the contribution of reflected flux to this patch is computed. This leads to double summation, over the source and over the receptor aperture. The amount of energy reflected from the source to the receptor is determined by the BRDF. Total flux $\Phi_{r}$ reflected from the material surface at a particular wavelength $\lambda$, reflected from patch at the surface area $d \mathbf{A}$ reaching detector aperture is computed by following formula:

$$
\begin{gathered}
\Phi_{r}=d \mathbf{A} \sum_{k} L\left(\mathbf{x} \leftarrow \Theta_{i_{k}}\right) \cos \left(\theta_{i_{k}}\right) \Delta \omega_{i_{k}} \times \\
\quad \times \sum_{j} f_{r}\left(\lambda, \mathbf{x}, \Theta_{i_{k}} \leftarrow \Theta_{r_{l}}\right) \cos \left(\theta_{r_{l}}\right) \Delta \omega_{d_{l}}
\end{gathered}
$$

where $d \mathbf{A}$ is area of a patch at the surface, $\Delta \omega$ (index $i$ refers to the source patches, index $r$ refers to the detector patches) is area of particular patch on the hemisphere, $\Theta$ is the direction from surface patch to the particular patch on the hemisphere, $\theta$ is angle between surface normal and that direction, see Figure 2. Function $f_{r}$ is BRDF and $L$ is incident radiance, where fragment of the radiant flux $\Phi_{r}$ is determined by the spectral energy of the light source. To acquire reflectance from above computation we divide the total flux by the irradiance:

$$
r=\frac{\Phi_{r}}{\sum_{k} L\left(\mathbf{x} \leftarrow \Theta_{i_{k}}\right) \cos \left(\theta_{i_{k}}\right) \Delta \omega_{i_{k}}} .
$$

The real measurement devices such as glossmeters can not measure the light reflection at a single point of the sample, but rather they measure reflection in a small region. Inhomogeneous surfaces such as metallic or pearlescent varnishes have varying $f_{r}$ at each surface point. This can happen often when there is a drawing on the surface, for example. It is therefore necessary to divide the sample into smaller parts and make a calculation in each of its part.

The primary standard for gloss measurements is a plane black glass having a refractive index of 1.567 at the wavelength $589.3 \mathrm{~nm}$.

The flux reflected off the black glass standard is computed by the following summation:

$$
\Phi_{\text {glass }}=d \mathbf{A} \sum_{k} L\left(\mathbf{x} \leftarrow \Theta_{i_{k}}\right) \cos \left(\theta_{i_{k}}\right) \Delta \omega_{i_{k}} F_{r}\left(\cos \theta_{i_{k}}\right),
$$

where $F_{r}$ is Fresnel reflection with the particular refractive index 1.567. We use complex refractive index $\hat{n}$ as a function of the wavelength. Equation 3 is derived from Equation 1 by incorporating $f_{r}$ of the black glass standard. Gloss units 
[Westlund and Meyer 2001] of particular material sample are then computed by

$$
G U=100 \frac{\Phi_{r}}{\Phi_{\text {standard }}} .
$$

We measured reflectance off real black glass standard using measuring device Konica MULTI GLOSS268. For the angle $20^{\circ}$ we got reflelectance $\hat{r}_{20^{\circ}}=0.045$, for the angle $60^{\circ}$ we got $\hat{r}_{60^{\circ}}=0.094$, angle $85^{\circ}$ produced reflectance $\hat{r}_{60^{\circ}}=0.61$. For comparison we also computed gloss units as the ratio of computed material reflectance (see Equation 2) and measured reflectance of black glass standard $\hat{r}$ multiplied by 100 :

$$
G U=100 \frac{r}{\hat{r}} .
$$

Our virtual measurement of black glass standard was used to find the correspondence with real measurement of black glass. We calibrate virtual gonio-spectrophotometer by setting the parameters that minimize the the root mean square error (RMSE) according to the real black glass measurements. We set the parameters and then we utilized our virtual gonio-spectrophotometer to perform computations of other samples.

Adjustable parameters of our virtual gonio-spectrophotometer are: angels to the detector and source aperture, size of the apertures and the refinement of the subdivided hemisphere. We simulated reflectance of the black glass standard using Equation 3. We performed simulations with variety of parameters setups. We set angles according mentioned standards to achieve correspondence with our real measurements of the black glass. Other parameters like aperture sizes and the degree of subdivision were varied. From the variety of setups we got one setup which produces minimal difference to the results acquired from real measurements of the black glass. We evaluated this difference in the terms of RMSE. By the minimization of this error we got virtual gonoi-spectrophotometer parameters that we will use to calibrate our virtual gonio-spectrophotometer for the purpose of virtual measurements.

\section{RESULTS}

Results are shown in Table II. Gloss units are computed using Equation 1, reflectance is computed using Equation 2. For the comparison we computed also gloss units using reflectance of real measured black glass standard as suggested in Section 4. Results for the case of $85^{\circ}$ are very small and thus seem unlikely. This suggests that proposed method is not suitable for gloss measurements under $85^{\circ}$. 


\begin{tabular}{|c|c|c|c|c|c|c|c|c|c|}
\hline & \multirow{2}{*}{\multicolumn{3}{|c|}{ reflectance }} & \multicolumn{6}{|c|}{ GU } \\
\hline & & & & \multicolumn{3}{|c|}{$\begin{array}{l}\text { computed by } \\
\text { the Eq. } 4\end{array}$} & \multicolumn{3}{|c|}{$\begin{array}{l}\text { computed by } \\
\text { the Eq. } 5\end{array}$} \\
\hline & $20^{\circ}$ & $60^{\circ}$ & $85^{\circ}$ & $20^{\circ}$ & $60^{\circ}$ & $85^{\circ}$ & $20^{\circ}$ & $60^{\circ}$ & $85^{\circ}$ \\
\hline steel & 0.04 & 0.08 & 0.03 & 89.41 & 81.366 & 4.87 & 88.9 & 85.1 & 4.9 \\
\hline $\begin{array}{c}\text { green } \\
\text { metallic paint }\end{array}$ & 0.0058 & 0.024 & 0.009 & 11.908 & 24.169 & 1.52 & 12.9 & 25.5 & 1.5 \\
\hline blue acrylic & 0.002 & 0.009 & 0.004 & 3.83 & 9.24 & 0.61 & 4,4 & 9.57 & 0.66 \\
\hline $\begin{array}{c}\text { gold } \\
\text { metallic paint }\end{array}$ & 0.003 & 0.01 & 0.008 & 5.41 & 11.9 & 1.22 & 6.67 & 10.64 & 1.3 \\
\hline black fabric & $1.741 \mathrm{E}-06$ & $5,356 \mathrm{E}-05$ & $9,128 \mathrm{E}-05$ & 0.0035 & 0.0535 & 0.0147 & 0.0038 & 0.0569 & 0.0149 \\
\hline
\end{tabular}

Table II. Computed gloss units and reflectance.

\section{CONCLUSION}

We examined the possibility of measuring gloss using data acquired by the digital camera. The pixels in each of the resulting images give measurements of light reflected from the surface. The known geometry of the surface, light source, and camera allows the incident and outgoing direction to be computed for each pixel. Each pixel in each image thus provides a measurement of the BRDF. Provided data served us as an input to the virtual gonio-spectrophotometer. Virtual gonio-spectrophotometer was set to measure gloss units according industrial standards Using this method we evaluated gloss units of multiple samples, mostly paint finishes. 


\section{REFERENCES}

523-89, A. D. 1999. Standard Test Method for Specular Gloss. ASTM.

Dorsey, J., Rushmeier, H., AND Sillion, F. 2008. Digital Modeling of Material Appearance. Morgan Kaufmann, San Francisco, USA.

Hunter, R. S. AND HAROLD, R. W. 1987. The Measurement of Appearance. John Wiley \& Sons, Inc., New York, NY, USA.

ISO2813. 1994. Paints and varnishes -Determination of specular gloss of non-metallic paint films at 20 degrees, 60 degrees and 85 degrees. Internation Organization for Standardization.

James A. Ferwerda, F. P. And GreenberG, D. P. 2001. A psychophysically-based model of surface gloss perception. In Proceedings SPIE Human Vision and Electronic Imaging '01. 291-301.

Matusik, W., Pfister, H., Brand, M., And McMillan, L. 2003. A data-driven reflectance model. In SIGGRAPH '03: ACM SIGGRAPH 2003 Papers. ACM, New York, NY, USA, 759-769.

MiHÁLIK, A. AND ĎURIKOVIČ, R. 2011. Virtual gonio-spectrophotometer for validation of BRDF designs. Central European Journal of Physics 9, 1334-1343.

Westlund, H. B. And Meyer, G. W. 2001. Applying appearance standards to light reflection models. In SIGGRAPH '01: Proceedings of the 28th annual conference on Computer graphics and interactive techniques. ACM, New York, NY, USA, 501-51.

Wills, J., Agarwal, S., Kriegman, D. J., And Belongie, S. J. 2009. Toward a perceptual space for gloss. ACM Trans. Graph. 28, 4.

Andrej Mihálik

Faculty of Mathematics, Physics and Informatics, Comenius University, 84248 Bratislava, Slovak Republic, http://www.fmph.uniba.sk email: mihalik@sccg.sk

\section{Roman Ďurikovič}

Faculty of Mathematics, Physics and Informatics, Comenius University, 84248 Bratislava, Slovak Republic, http://www.fmph.uniba.sk email: roman.durikovic@fmph.uniba.sk 